\title{
A temática da Rede Cegonha e a inserção do enfermeiro nesse contexto
}

\author{
The theme of the "Stork Network" and the inclusion of nurses in this context
}

Edith Monteiro de Oliveira*, Denize Duarte Celento

Como citar esse artigo. Oliveira EM, Celento DD. A temática da Rede Cegonha e a inserção do enfermeiro nesse contexto. Revista de Saúde. 2016 Jan./Jun.; $07 \quad$ (1): 33-38.

\begin{abstract}
Resumo
Esta pesquisa justifica-se em função da análise de conhecimento da rede de atenção à Saúde vigente, em busca de um novo padrão capaz de melhorar o atual modelo de atenção obstétrica. O objetivo principal é descrever a Rede Cegonha e analisar o papel do enfermeiro nesse contexto. Foi realizada uma revisão bibliográfica de publicações entre 2010 e 2014, na base de dados BIREME, e legislações por órgãos reguladores da área da Saúde. A Rede Cegonha prevê ações para a melhoria do acesso e da qualidade da assistência à mulher e da criança, por meio de ações inseridas em quatro componentes fundamentais da estratégia, que são: pré-natal; parto e nascimento; puerpério e atenção integral à Saúde da criança e sistema logístico de transporte, sanitário e regulamentação. A estratégia busca uma mudança visionária em relação ao modelo de atenção ao parto e nascimento praticado no país, valorizando o profissional de Enfermagem que participa de todos os níveis de assistência, viabilizando o acesso, o acolhimento e a resolubilidade, de forma a assegurar à mulher seu direito de ser protagonista do processo parturitivo.
\end{abstract}

Palavras-chave: Cuidado Pré-Natal; Parto; Nascimento; Enfermeira.

\begin{abstract}
This research is based on the analysis and knowledge of the current network of care and health, in search of new standards capable of improving the current model of obstetric care. The general purpose was to describe the Stork Network and analyze the role of the nurse in this context. A literature review of publications between 2010 and 2014, found at BIREME database, and legislation by health regulatory agencies was conducted. The Stork Network provides actions for improving access and quality of care for women and children, through actions inserted into four fundamental components of the strategy: prenatal, labor and childbirth, puerperium and integral attention to children's health, and logistics, transportation and regulatory systems. The strategy seeks a visionary change from the current care model practiced in the country regarding labor and birth, valuing the Nurse participating in all levels of care, enabling access, acceptance and resolution, to ensure the woman her right to be protagonist in the birth process.
\end{abstract}

Keywords: Prenatal Care; Childbirth; Nurse.
Este estudo está alicerçado no conceito ampliado de saúde - "A saúde é um direito fundamental do ser humano, devendo o Estado prover as condições indispensáveis ao seu pleno exercício." (Art. $2^{\circ}$ - Lei $\mathrm{n}^{\circ}$ 8.080). Desse modo, a assistência deve se pautar no princípio da integralidade, entendido como "conjunto articulado e contínuo das ações e serviços preventivos e curativos, individuais e coletivos, exigidos para cada caso em todos os níveis de complexidade do sistema." Nesse sentido, a realidade brasileira aponta para uma reestruturação da atenção à saúde, enfatizando um avanço das políticas de saúde da mulher e da criança, visando implementar estratégias objetivando a melhoria da qualidade da atenção materno-infantil, perfazendo os seguintes itens: pré-natal, parto e nascimento, puerpério e atenção integral à saúde da criança.

Em 1990, a taxa de mortalidade materna foi de 140 óbitos por 100.000 nascidos vivos. Já em 2007, verificou-se 75 óbitos por 100.000 nascidos vivos. Embora essa taxa tenha sofrido uma redução de 52\% no período descrito acima, o número de mortes maternas no Brasil permanece elevado. ${ }^{2}$ Segundo o Ministério da Saúde, a partir de 2001, houve o decréscimo na fugacidade do indicador da mortalidade materna. No entanto, a Organização das Nações Unidas, propôs na Cimeira do Milênio realizada na cidade de Nova Iorque, em setembro de 2000, oito objetivos denominados "Objetivos do Milênio" e, de acordo com o exposto acima, são necessários maiores esforços para atingir valor igual ou inferior a 35 óbitos por 100.000 nascidos vivos até 2015, meta determinada no quinto Objetivo do Milênio (ODM) de melhorar a saúde das gestantes.

\footnotetext{
Afiliação dos autores: Universidade Severino Sombra, Pró-Reitoria de Ciências da Saúde e Humanas, Curso de Enfermagem, Vassouras-RJ, Brasil.

* Endereço para correspondência: Universidade Severino Sombra, Av. Exped. Oswaldo de Almeida Ramos, 280 - Centro - Vassouras, RJ - CEP $27700-000$. E-mail: enf.editholiveira@outlook.com
} 
No que concerne à mortalidade infantil, verificouse uma redução de cerca de $60 \%$ desses óbitos nas últimas duas décadas. Em 1990, verificou-se 57,1 óbitos por 1.000 nascidos vivos e em 2008 o número reduziu para 19,0 óbitos por 1.000 nascidos vivos. ${ }^{2}$ A redução da taxa de mortalidade é sabidamente um indicador clássico de saúde por refletir as condições ambientais e socioeconômicas da população, todavia, a mortalidade infantil é também identificada como um acontecimento indicador da facilidade de acesso e da qualidade dos serviços. Os resultados indicam que o Brasil cumprirá o quarto ODM que visa a redução em dois terços de mortalidade na infância até 2015 .

As intervenções públicas para aperfeiçoar a melhoria ao acesso e a qualidade da assistência prénatal, parto e ao recém-nascido têm sido consideradas intervenções complexas e custosas. A partir do entendimentodequeamorbimortalidadematerno-infantil são eventos complexos e, portanto, multifatoriais, essas questões permanecem um desafio para o Brasil. Alguns fatores têm dificultado a melhoria desses indicadores, tais como: o financiamento insuficiente; a deficiente regulamentação do sistema de saúde; a fragmentação das ações e dos serviços de saúde; a organização dos serviços (incluindo a gestão do trabalho das equipes de saúde); e, ainda, a produção do cuidado, que tende a "medicalizar" e "intervir" desnecessariamente nos processos de gestação, parto e nascimento. ${ }^{3}$

Por todos esses motivos apresentados, diversas ações de saúde vêm sendo direcionadas aos segmentos materno-infantis de forma mais sistemática no Sistema Único de Saúde (SUS), como parte dos esforços intergovernamentais para redução dos indicadores nestes segmentos populacionais. ${ }^{4} \mathrm{Um}$ exemplo dessas ações é a criação das Redes de Atenção à Saúde, pela Portaria 4.279 de 30 de dezembro de 2010, que estabelece diretrizes para sua organização, visando estrategicamente solucionar a fragmentação da atenção e aperfeiçoar o funcionamento político-institucional do SUS, aspirando proporcionar ao usuário um serviço de qualidade com eficiência e efetividade. ${ }^{2}$ Consoante com o compromisso em relação à melhora da qualidade de vida de mulheres e crianças, foi lançado em 2004, pela Presidência da República, após aprovação na Comissão Intergestores Tripartite (CIT) e no Conselho Nacional de Saúde, o Pacto Nacional pela Redução da Mortalidade Materna e Neonatal. Em 2005, afirmando-se como política de Estado, foi composta a Comissão Nacional de Monitoramento e Avaliação da Implementação do Pacto Nacional pela Redução da Mortalidade Materna e Neonatal. ${ }^{5}$

Os objetivos prioritários e essenciais no Sistema Único de Saúde e desenvolvidos no atendimento básico à saúde e pelo Programa Saúde da Família (PSF), foram adotados oficialmente a partir de 2000, quando foi lançado o Programa de Humanização do Pré-Natal e
Nascimento (PHPN) de acordo com a portaria 569/GM/ MS que objetiva o resgate da atenção obstétrica integrada, qualificada e humanizada com o envolvimento de forma articulada dos Estados, Municípios e das unidades de saúde nestas ações, visando assim garantir o acesso da gestante aos serviços de saúde para assistência pré-natal, intercorrências na gravidez e para o parto. ${ }^{6}$

Segundo o Ministério da Saúde, em 2007, o prénatal alcançou $89 \%$ de cobertura, e a captação precoce das gestantes foi de $83,6 \%$ (primeira consulta do pré-natal no primeiro trimestre). No entanto, as mortes causadas por síndromes hipertensivas e por causas perinatais e a incidência de sífilis congênita evidenciam a necessidade de qualificar o pré-natal. Outra questão a ser abordada, é o elevado número de partos cirúrgicos (cesáreas) no Brasil, em relação a outros países. Os percentuais revelam que as cesáreas aumentaram de 40\%, em 1996, para 46,5\% em 2007, sendo necessária, a elaboração de medidas que visem potencializar a percepção feminina sobre a vivência do parto e reduzir assim intervenções obstétricas eletivas. $^{7}$

Diante disso, este artigo justifica-se em função da análise de conhecimento da Rede de Atenção à Saúde vigente, buscando a existência de um novo padrão capaz de realizar mudanças no atual modelo de atenção obstétrica, garantindo assim um atendimento humanizado e seguro às gestantes, tornando-as protagonistas do processo de gestação e parto.

A investigação da saúde da mulher no atual cenário brasileiro e o pleito em relação às perspectivas dos pontos de atenção necessários ao cuidado maternoinfantil, conectados à Rede de Saúde foram motivadores e determinantes para a realização deste projeto de pesquisa.

Como profissional de enfermagem, que possui a ciência pautada no cuidado, cuidar é mais que um ato; é uma atitude que envolve mais que um momento de atenção, de zelo e cuidado, importando uma atitude de ocupação, preocupação, de responsabilização com o outro. Contudo, o conceito e os objetivos da Rede Cegonha, lançada em março de 2011 e instituída pela Portaria ${ }^{\circ} 1.459$, de 24 de junho de 2011, apontada como uma estratégia do Ministério da Saúde que preconiza assegurar, à mulher e à criança, o direito à atenção humanizada durante o pré-natal, parto e nascimento, puerpério e atenção infantil em todos os serviços de saúde do Sistema Único de Saúde (SUS). ${ }^{8}$

A Rede Cegonha prevê ações para a melhoria do acesso e da qualidade da assistência à mulher e da criança, por meio da vinculação da gestante à unidade de referência e o transporte seguro, e da implementação de boas práticas na atenção ao parto e nascimento, incluindo o direito ao acompanhante de livre escolha da mulher no parto. Estas ações estão inseridas em quatro componentes estruturantes da estratégia, que são: prénatal, parto e nascimento, puerpério e atenção integral à 
saúde da criança e sistema logístico, transporte sanitário e regulação.

Da inquietação como enfermeira, surgiu a busca pelo comportamento do profissional nesse cenário, que almeja a mudança cultural e o realinhamento das ações.

As questões que nortearam este estudo abrangem os problemas enfrentados pelos formuladores federais para criação da Rede Cegonha e as metas que a mesma almeja alcançar, diante da estrutura do seu funcionamento.

O objetivo geral do estudo visa descrever a Rede Cegonha e o papel ou influência do enfermeiro nesse contexto, Como objetivos específicos: delinear os componentes da Rede Cegonha; identificar as ações de atenção à saúde compreendida em cada componente da Rede; analisar a operacionalização da Rede Cegonha.

\section{Materiais e Métodos}

Foi realizada uma pesquisa exploratória e levantamento bibliográfico de publicações científicas de Enfermagem com relação à importância e papel do enfermeiro no gerenciamento e qualidade das unidades hospitalares, utilizando-se a base de dados BIREME.

\section{Resultados}

A análise documental de publicações entre 2010 e 2014, na base de dados BIREME produziu 279 artigos, dentre os quais 11 foram selecionados e analisados nesse estudo.

\section{Discussão}

ARede Cegonha foi desenvolvida e fundamentada em ações a fim de reduzir a mortalidade maternoinfantil no país a partir de tratados internacionais, entre eles, os Objetivos de Desenvolvimento do Milênio, e de experiências existentes em ações para a saúde das mulheres e crianças no país, que seguem um modelo marcado por medicalização, intervenções desnecessárias e potencialmente iatrogênicas, assim como a prática abusiva de cesarianas e o desrespeito à autonomia e aos direitos da gestante, muitas dessas de baixa escolaridade e vulneráveis socialmente. ${ }^{9}$

Justifica-se o exposto à incapacidade institucional do SUS, caracterizado por ser uma rede fragmentada e pouco resolutiva devido à incapacidade gerencial e de alguns membros da equipe, além da inadequação de ambiência, estrutura e equipamentos de serviços de saúde, do financiamento insuficiente e a ineficiência de alocação de recursos públicos. De acordo com o Ministério da Saúde, são fatores que tem dificultado a melhoria dos indicadores de cunho materno-infantil: o financiamento insuficiente; a deficiente regulação do sistema de saúde; a fragmentação das ações e dos serviços de saúde; a organização dos serviços (incluindo a gestão do trabalho das equipes de saúde); e, ainda, a produção do cuidado, que tende a "medicalizar" e "intervir" desnecessariamente nos processos de gestação, parto e nascimento. ${ }^{8}$

Todavia, o elevado número de gravidezes indesejadas, a dificuldade de acesso das gestantes ao pré-natal de qualidade, a peregrinação das gestantes no momento do parto e a prática de partos e nascimentos inadequados, e sem base em evidências científicas, foram problemáticas e visualizadas pelos formuladores federais e executores para a elaboração de um novo modelo de assistência materno-infantil visando a melhoria ao acesso e a qualidade da assistência prénatal, parto, ao recém-nascido e a criança até os vinte e quatro meses. ${ }^{10}$

Diante do cenário apresentado, destaca-se que a rede de atenção obstétrica a ser instituída em todo o território brasileiro, tem um formato próprio, alicerçando seus processos e ações em indicadores estratégicos reais e a partir da avaliação desses indicadores a pactuação de metas municipais com as equipes da Rede, monitorando periodicamente e sistematicamente, buscando assim, atingir os objetivos estratégicos que conduzam à redução da morbimortalidade materno-infantil, conforme priorizado pelos "Objetivos do Milênio".

A Atenção Primária à Saúde, responsável pelo atendimento à população e o principal canal de comunicação das Redes de Atenção à Saúde, incorpora as ações da Rede Cegonha e se responsabiliza pelos componentes Pré-Natal, Puerpério e Atenção Integral à Saúde da Criança, sendo potencialmente ativa e fundamentada no cuidar como processo integrante da humanização nas Unidades Básicas de Saúde, o que a torna satisfatória na busca pela efetivação da Rede Cegonha, tendo em vista os quase $100 \%$ dos municípios brasileiros aderidos ao componente pré-natal. Em conformidade, apresenta-se o Programa Nacional de Melhoria do Acesso e da Qualidade de Atenção Básica (PMAQ-AB), outro elemento positivo que tem dentro de suas avaliações algumas ações da Rede Cegonha.

As Unidades Básicas de Saúde por meio do SISPRENATAL identificarão as gestantes que terão direito ao apoio financeiro pautado no fato de que cada gestante receberá dois valores diferenciados em momentos distintos: no primeiro trimestre, posteriormente a entrega do resultado do exame de VDRL (Venereal Disease Research Laboratory), e no terceiro trimestre, após a entrega do terceiro exame de VDRL.

No componente Pré-natal, o objetivo estratégico compreende a garantia do acolhimento, a ampliação do acesso e a melhoria da qualidade do Pré-Natal por 
meio de ações que visam qualificar os profissionais da Atenção Básica, ofertar teste rápido de gravidez, financiar os exames de pré-natal, ofertar o teste rápido de sífilis, disponibilizar a bolsa Rede Cegonha e o kit de atenção pré-natal para as Unidades Básicas de Saúde.

A literatura concorda que há fragilidades no que tange à integralidade e humanização na atenção obstétrica no processo parturitivo, principalmente quanto ao vínculo e acolhimento, o uso indiscriminado de tecnologias e intervenções desnecessárias, conferindo assim a vulnerabilidade da mulher nesse contexto. Sendo necessário um acompanhamento por profissionais qualificados, perfazendo o saber clínico e se corresponsabilizando, se conscientizando, se engajando verdadeiramente como profissionais de saúde e almejando a promoção do cuidado na estruturação de projetos terapêuticos singulares. ${ }^{11-13}$

Entretanto, o enfermeiro deve estar apto e qualificado a diferentes casos e situações do meio que venham a surgir, por exemplo, a constatação de HIV ou Sífilis em mulher consideradas vulneráveis, conforme apresenta Carneiro ${ }^{14}$, que abordou a dificuldade profissional em atuar com a real complexidade do fenômeno HIV/AIDS, postergando enfrentamentos e os profissionais ao lidar com os medos das usuárias também desafiam os seus próprios medos. Nessa perspectiva, verifica-se a necessidade de trabalhar com uma equipe multiprofissional inserida tanto no contexto da Rede Primária a Rede Terciária, atendendo de forma consolidada tanto os profissionais que lidam com as gestantes em questão quanto as próprias gestantes, visando a presteza de um serviço qualificado. ${ }^{15}$

A promoção do aleitamento materno até os dois anos de idade, considerando o aleitamento exclusivo nos seis primeiros meses, e introduzindo alimentos complementares saudáveis em tempo oportuno, fortalece a Rede Amamenta e Alimenta Brasil, também são ações estabelecidas no contexto da Rede Cegonha; organizando os serviços de atenção primária para garantir o acompanhamento da criança de maneira a avaliar o crescimento e desenvolvimento em todas as consultas de rotina, garantindo acesso às vacinas disponíveis no SUS e o preenchimento adequado da Caderneta de Saúde da Criança, atendendo resolutivamente o surgimento de possíveis intercorrências. ${ }^{16-19}$

Em nível secundário e terciário, o Ministério da Saúde de acordo com a lógica da Rede Cegonha, priorizou estrategicamente o planejamento e $\mathrm{o}$ investimento em ações que visam o financiamento de obras, a ambiência nas maternidades e hospitais, o aumento dos custeios dos serviços de saúde e recursos para a aquisição de equipamentos. Além da criação, regulamentação e financiamento de dispositivos que incentivam o parto humanizado, a qualificação do SAMU com equipagem de urgência ao neonato, o financiamento e ampliação dos leitos de UTI adulto e neonatal, UCI Convencional e Canguru.

A garantia de vinculação da gestante à unidade de referência e de transporte segura, se dará por meio de ações estratégicas que concedam o vale-transporte para gestantes realizarem consultas de pré-natal e exames necessários, vale-táxi para gestantes se deslocarem ao serviço de saúde durante o trabalho de parto. Com os Centros de Parto Normal, a atuação do enfermeiro obstetra ocorrerá de forma a aumentar a satisfação das parturientes a partir da experiência vivenciada, viabilizando segurança e considerando tanto os aspectos fisiológicos, quantos os psicológicos e todo o contexto sociocultural, diminuindo o uso de intervenções obstétricas e buscando assim a melhoria dos indicadores de morbimortalidade materno-infantil.

Em conformidade, a Resolução do COFEN $n^{0} 223 / 1999^{20}$ (revogada em $2015^{21}$ ) que faz referência a atuação dos profissionais de Enfermeiros na Assistência à Mulher no Ciclo Gravídico Puerperal, o Ministério da Saúde reporta-se ao trabalho do profissional de Enfermagem no contexto dos Centros de Parto Normal, a realização de parto normal sem distócia, assistindo à gestante, parturiente e puérpera e acompanhando todo o trabalho de parto e intervindo quando necessário e em consonância com a sua capacitação técnico-científica, priorizando procedimentos que julgar imprescindíveis para a segurança do binômio mãe-filho.

Nessa concepção, observa-se outro ponto forte da Rede Cegonha que promove a capacitação e a qualificação dos profissionais de saúde, valorizando o enfermeiro da Rede de Atenção Básica e o enfermeiro obstetra/obstetriz, para que este possa agir de forma a prestar um serviço humanizado e de qualidade às mulheres e as crianças, dando uma resposta à violência obstétrica através do cuidado e assistência baseada em evidências. ${ }^{22}$ As ações do Ministério da Saúde vêm intensificando a formação e a especialização de profissionais com este perfil paraatuarem dentro do SUS junto à estratégia da Rede Cegonha.

Quanto à estrutura operacional da Rede Cegonha que potencializa suas ações, caracteriza-se pela incorporação de Sistemas Logísticos já existentes no Sistema Único de Saúde, que perfaz o registro eletrônico em saúde, prontuário clínico, o cartão de identificação, os sistemas de acesso regulado a atenção com o tema "Garantia de Vaga Sempre" para bebês e gestantes, garantindo a eficiência do atendimento a gestante, parturiente e recém-nascido e os sistemas de transporte em saúde meio facilitador do acesso, sendo este direito dos usuários do SUS.

Firmado na experiência do SUS que em seus 26 anos de existência apoderam-se de instrumentos normativos, de monitoramento e de avaliação considerados consistentes e consubstanciado. Paulatinamente, o modelo de gestão federativo induz a contratualização e responsabilização da União, 
Estados e Municípios, favorecendo assim a existência de Conselhos, Comissões Inter gestoras, além de outros dispositivos de participação do SUS, beneficiando assim o respeito e a valorização democrática.

Conquanto, o modelo de atenção da Rede Cegonha propõe uma mudança na lógica do cuidado, que objetiva atender de forma continuada e integrada, desde o nível primário ao terciário, executando congruentemente a humanização, o respeito à fisiologia do parto e ao protagonismo da mulher, dissipando com a ideia da hegemonia hospitalar e desmistificando o papel do médico como facilitador da redução da mortalidade materno-infantil.

A mulher na sociedade tinha função primordial de reprodução e a organização dos serviços de saúde foi desenvolvida conforme essa percepção, menosprezando outros ângulos da vida das mulheres, nesse sentido a hierarquização e os diferentes papéis exercidos por ambos os gêneros na sociedade influenciaram diretamente nas políticas de saúde.

Nessa perspectiva, Carneiro ${ }^{13}$ salienta que no Brasil, a saúde da mulher foi incorporada às políticas públicas de saúde nas primeiras décadas do século XX, sendo, nesse período, limitada às demandas relativas à gravidez e ao parto. Os programas materno-infantis implantados nesse período reduziam seu papel social à função reprodutiva, vendo a mulher apenas como mãe e cuidadora da saúde da família.

O advento de políticas de saúde voltadas para a mulher, acentuou a hospitalização do parto, propiciando a medicalização, o controle do período gravídico puerperal e o parto que antes era visto como um processo natural, familiar e privativo, tornou-se vivencia pública, com a presença de diferentes atores protagonizando esse período. Tal evento apresentado favoreceu a submissão da mulher deixando-a de ser protagonista do processo parturitivo.

Bussanello $^{23}$ considera que as mulheres estão expostas às determinações das políticas públicas de saúde e que as propostas e os programas voltados para essa população enfatizam o respeito à autonomia da mulher. Contudo, ao seremcolocadas em prática, nos diversos níveis de atenção, as mulheres passam a ser objeto dessas políticas, assim como, os enfermeiros, frequentemente, estão submissos às suas normas e diretrizes. A cobrança da chefia pelo cumprimento de metas assistenciais, muitas vezes, faz com que a opinião das mulheres não seja ouvida pelos enfermeiros.

Nesse sentido, Carneiro ${ }^{13}$ associa que a negação do princípio da autonomia traduz a incidência do poder dos profissionais de saúde sobre as mulheres e se tratando desses, a verdade é representada pelo conhecimento científico do qual se dispõem e que através dele, se estabelece não só a diferenciação entre as categorias sociais detentoras e não detentoras do saber, mas também se exerce o poder das primeiras em relação às últimas, estas representadas pelos (as) usuários (as).

Em reconhecimento ao exposto, a Rede Cegonha lançada pelo Ministério da Saúde objetiva organizar e estruturar a atenção à saúde maternoinfantil, pautada nos princípios do SUS que preconiza a descentralização, humanização, integralidade e equidade, garantindo à gestante autonomia para ser protagonista do processo de gestação, parto e puerpério, acolhendo-a desde a sua captação para a realização do pré-natal na Unidade Básica de Saúde até o puerpério, bem como à criança,garantindo um nascimento seguro, ao crescimento e ao desenvolvimento saudáveis, com assistência de qualidade e humanizada, proporcionada pela capacitação dos profissionais de saúde.

\section{Declarações}

Os autores não possuem conflitos de interesse diretos ou indiretos. A fonte de financiamento deste estudo não foi declarada.

\section{Referências}

1. Brasil, Ministério da Saúde. Lei n 8.080, de 19 de setembro de 1990. Dispõe sobre as condições para a promoção, proteção e recuperação da saúde, a organização e o funcionamento dos serviços correspondentes e dá outras providências. n.d. [citado em 14 de setembro de 2014]; Disponível em: http://portalsaude.saude.gov.br/

2. Brasil, Ministério da Saúde. Portaria n ${ }^{4}$ 4.279/GM/MS, de 30 de dezembro de 2010. Estabelece diretrizes para a organização da Rede de Atenção à Saúde no âmbito do Sistema Único de Saúde (SUS). n.d. [citado em 14 de setembro de 2014]; Disponível em: bvsms.saude.gov.br/bvs/saudelegis/ gm/2010/prt4279 $30 \quad 12$ 2010.html/

3. Brasil, Ministério da Saúde. Portaria No 650, de 5 de outubro de 2011 Dispõe sobreos Planos de Ação regional e municipal da Rede Cegonha. Brasília (DF). 2011a [citado em 14 de setembro de 2014]; Disponível em: http://bvsms.saude.gov.br/bvs/saudelegis/sas/2011/prt0650 $05 \quad 102011$. html

4. Brasil, Ministério da Saúde. Secretaria de Atenção à Saúde. Manual prático para implementação da Rede Cegonha. Brasília (DF). 2011b [citado em 14 de setembro de 2014]; Disponível em:www.saude.mt.gov.br/arquivo/3062

5.Brasil, Ministério da Saúde. Lei ${ }^{\circ} 11.108$, de 07 de abril de 2005; Do Subsistema de Acompanhamento durante o trabalho de parto, parto e pósparto imediato. n.d. [citado em 14 de setembro de 2014]; Disponível em: http://portalsaude.saude.gov.br/

6. Brasil, Ministério da Saúde. Portaria n569/GM/MS, de 01 de junho de 2000; Programa de Humanização no Pré-natal e Nascimento. n.d. [citado em 14 de setembro de 2014]; Disponível em:sisprenatal.datasus.gov.br SISPRENATAL/Portaria 569 GM.PDF

7. Brasil, Ministério da Saúde. Lei $n^{\circ} 11.634$, de 27 de dezembro de 2007; Dispõe sobre o direito da gestante ao conhecimento e a vinculação à maternidade onde receberá assistência no âmbito do Sistema Único de Saúde. n.d. [citado em 14 de setembro de 2014]; Disponível em:http://portalsaude. saude.gov.br/

8. Brasil, Ministério da Saúde. Secretaria de Atenção á Saúde. Departamento de Ações Programáticas Estratégicas. Portaria N $\mathrm{N}^{\mathrm{o}} 1.459 / \mathrm{GM}, 24$ de junho de 2011. Institui, no âmbito do Sistema Único de Saúde, a Rede Cegonha. Brasília (DF). 2011c[citado em 14 de setembro de 2014]; Disponível em: http://bvsms.saude.gov.br/bvs/folder/departamento_acoes_programaticas estrategicas dapes.pdf

9.Leal MDC, Gama SGND. Nascer no Brasil. Cad Saúde Pública [online]. 2014;30(Sup.1):S5-S. 
10. Albuquerque RA, Jorge MSB, Franco TB, Quinderé PHD. Produção do cuidado integral no pré-natal: itinerário de uma gestante em uma unidade básica de saúde da família. Interface (Botucatu) [online]. 2011;15(38):67786.

11. Pinheiro BC, Bittar CML. Expectativas, perceções e experiências sobre o parto normal: relato de um grupo de mulheres. Fractal : Revista de Psicologia. 2013;25(3):585-602.

12. Cabral FB, Hirt LM, Van der Sand ICP. Atendimento pré-natal na ótica de puérperas: da medicalização à fragmentação do cuidado. Revista da Escola de Enfermagem da USP. 2013;47(2):281-7.

13Líbera BD, Saunders C, Santos MMAdS, Rimes KA, Brito FRdSdS, Baião MR. Avaliação da assistência pré-natal na perspectiva de puérperas e profissionais de saúde. Ciênc saúde coletiva [online]. 2011;16(12):4855-64.

14.Carneiro AJS, Coelho EdAC. Aconselhamento na testagem anti-HIV no ciclo gravídico-puerperal: o olhar da integralidade. Ciênc Saúde coletiva [online]. 2010;15(Sup.1):1216-26.

15. Brasil, Ministério da Saúde. Secretaria de Vigilância em Saúde. Departamento de DST, Aids e Hepatites Virais. Guia orientador para a realização das capacitações para executores e multiplicadores em Teste Rápido para HIV e Sífilis e Aconselhamento em DST/Aids na Atenção Básica para gestantes/Aids / Ministério da Saúde, Secretaria de Vigilância em Saúde, Departamento de DST, Aids e Hepatites Virais. 2013 [citado em 14 de setembro de 2014]; Disponível em: http://dab.saude.gov.br/portaldab/ ape_redecegonha.php?conteudo=teste_rapido_balancas

16. Brasil,. Ministério da Saúde. Secretaria de Atenção à Saúde. Departamento de Atenção Básica. Manual instrutivo das ações de alimentação e nutrição na Rede Cegonha [recurso eletrônico] / Ministério da Saúde. Secretaria de Atenção à Saúde. Departamento de Atenção Básica. - Brasília: Ministério da Saúde, 2013. 32 p. Disponível em: http://189.28.128.100/dab/docs/portaldab/ publicacoes/manual_alimentacao_nutricao_rede_cegonha.pdf
17.Campos ABF, Pereira RA, Queiroz J, Saunders C. Ingestão de energia e de nutrientes e baixo peso ao nascer: estudo de coorte com gestantes adolescentes. Rev Nutr [online]. 2013;26(5):551-61.

18. Machado MMT, Braga MQC, Galvão MTG. Problemas com a mama puerperal revelados por mães soropositivas. Revista da Escola de Enfermagem da USP. 2010;44(1):120-5.

19. Morgado CMDC, Werneck GL, Hasselmann MH. Rede e apoio social e práticas alimentares de crianças no quarto mês de vida. Ciênc saúde coletiva [online]. 2013;18(2):367-76

20. COFEN. Resolução COFEN-223/1999 -Dispõe sobre a atuação de Enfermeiros na Assistência à Mulher no Ciclo Gravídico Puerperal. 1999 [citado em 14 de setembro de 2014]; Disponível em: http://www.cofen.gov. br/resoluo-cofen-2231999_4266.html

21. COFEN. Resolução Cofen $n^{\circ}$ 477/2015 - Dispõe sobre a atuação de Enfermeiros na assistência às gestantes, parturientes e puérperas. 1999 [citado em 14 de setembro de 2014]; Disponível em:http://www.cofen.gov. br/resolucao-cofen-no-04772015_30967.html

22.Silva AGE, Moraes CL, Reichenheim ME. Violência física entre parceiros íntimos: um obstáculo ao início do acompanhamento da criança em unidades básicas de saúde do Rio de Janeiro, Brasil? Cad Saúde Pública [online]. 2012;28(7):1359-70.

23. Busanello J, Lunardi Filho WD, Kerber NPdC, Lunardi VL, Santos SSd. Participação da mulher no processo decisório no ciclo gravídico-puerperal: revisão integrativa do cuidado de enfermagem. Rev Gaúcha Enferm [online]. 2011;32(4):807-14 